\title{
Bio Efficacy and Effect on Natural Enemies of Acaricide Etoxazole 10 SC against Mite Pests of Apple in Kashmir
}

\author{
Asma Sherwani ${ }^{1}$, Peerzada Shafat Hussian ${ }^{2}$ and Malik Mukhtar ${ }^{2} *$ \\ ${ }^{1}$ Division of Entomology Faculty of Horticulture, ${ }^{2}$ Krishi Vigyan Kendra Bandipora \\ Sher-e-Kashmir University of Agricultural Sciences and Technology of Kashmir \\ Shalimar Srinagar 190025 J\&K, India \\ *Corresponding author
}

\section{A B S T R A C T}

\section{Keywords \\ Natural enemies, \\ Acaricide, \\ Etoxazole, Mite pests, Apple \\ Article Info \\ Accepted: \\ 20 January 2020 \\ Available Online: \\ 10 February 2020}

Acaricide Etoxazole 10 SC was tested against European red mite Panonychus ulmi (ERM) and two-spotted spider mite Tetranychus ulmi (TSSM) for bio efficacy, phytotoxicity and effect on natural enemies in apple orchards at two locations in Kashmir during the year 2018 and 2019. Propargite was used as standard check for conducting the experiment. Both the acaricides were sprayed during the successive summer seasons to suppress different stages of the pests. Etoxazole was sprayed at the concentrations of $0.2,0.5,0.8$ and $1.1 \mathrm{ml} \mathrm{water}^{-1}$. All the concentrations significantly reduced the populations of both the mite pests over control. Highest cumulative mean percent mortality of 70.62 and 70.0 were recorded at the concentration of $1.1 \mathrm{ml}^{\text {water }}{ }^{-1}$ against European red mite at locations of Nagbal and Chandpora respectively. Similarly highest cumulative mean percent mortality of 69.11 and 69.17 were recorded at the concentration of $1.1 \mathrm{ml}^{\text {water }}{ }^{-1}$ against twospotted spider mite at locations of Nagbal and Chandpora respectively. Propargite @ $2 \mathrm{ml}$ water $^{-1}$ exhibited cumulative mean percent mortality of 72.80 and 73.34 against European red mite and 72.32 and 71.58 against two spotted spider mite at locations of Nagbal and Chandpora respectively. None of the phytotoxic effect was seen on the apple trees at each of the concentration tested. Natural enemies like Amblyseius and Coccinellids were the key predators found feeding on European red mite and two-spotted mite respectively. Among the various concentrations of Etoxazole, maximum cumulative mean percent mortality of natural enemies was found at the concentration of $1.1 \mathrm{ml} \mathrm{water}^{-1}$.

\section{Introduction}

Apple Malus domestica (Borkh) is remarkably predominant among all other temperate fruits in Jammu and Kashmir. However, the production and quality of apple is affected by a number of serious pests causing heavy economic loss to the crop. The major insect pests attacking this crop are San Jose scale, European red mite, two spotted spider mite and apple wooly aphid. Among all these pests European red mite and two-spotted spider mite have established themselves as a serious pest of apple trees in Jammu and Kashmir (Anonymous, 2014, Bhalla and Gupta, 1993). Since these pests have been accidentally 
introduced to many countries and are considered major pests in most regions of the world where deciduous fruits are grown (Madson and Morgan, 1970), their management is of paramount importance to contain their spread. The damage by European red mite, Panonychus ulmi (Koch) is caused by nymphs and adults, which suck the sap from leaves, weakens the plant and leaves thereby reducing the yield of the plant.

Etoxazole a systemic acaricide is used for the management of red spider mites. It attacks a number of mites in the egg, larval and nymphal stages. The mode of action was originally believed to disrupt the molting process but has since been shown to inhibit chitin synthesis. Keeping in view the novel nature of this acaricide under Kashmir conditions and severity of the pests, an experiment was undertaken to test the bio efficacy of Etoxazole, its phytotoxicity, impact on quality of apple yield and effect on natural enemies in the apple ecosystem of Kashmir valley.

\section{Materials and Methods}

To evaluate the effectiveness of Etoxazole against European red mite and two-spotted mite on apple, field trial was carried out in the farmer's holding apple orchard at Nagbal and Chandpora locations of Ganderbal and Srinagar district respectively of Kashmir during the year 2018 and 2019. The orchard had apple trees of Red Delicious cultivar having uniform size and age of 15 years. The orchard was selected taking into account the outbreak and damage caused by the mite pests. The experiment was laid in a randomized block design (RBD) with six treatments with three replications each. Four treatments of Etoxazole 10 SC @ $0.2 \mathrm{ml}, 0.5 \mathrm{ml}, 0.8 \mathrm{ml}$ and $1.1 \mathrm{ml} \mathrm{water}^{-1}$ were compared with standard check Propargite @ $2 \mathrm{ml}$ water $^{-1}$ in successive summer seasons. Water sprayed apple trees were kept as control. Spraying was done by high volume power sprayer @ 15 liter spray solution per tree. Pre-treatment count of the mite population was recorded one day before the treatment. Post treatment observations were recorded after $3,7,10,14,21$ and 28 days post spray for the active populations of ERM and TSSM. The pest counts were recorded using hand lens during morning hours. The observations on ERM and TSSM were recorded from 20 leaves /tree from different directions and different heights (bottom, middle and top) for observation on adult and immature stages. Bio efficacy and phytotoxicity observations were also recorded after spray at both the locations. Phytotoxicity data such as leaf injury on tips/surface, yellowing, wilting, necrosis, hyponasty and epinasty were also recorded at different days post spray using (1-10) scale. Yield was calculated on the basis of A grade apple boxes /tree/treatment at harvesting time. Similarly the observations on natural enemies were also recorded one day before the spray (pre treatment count) and 1, 3, 7 and 15 days post treatment for each replication. The percent reduction in ERM and TSSM over control was calculated using the following formula (Henderson and Tilton 1955).

Percent reduction $=$

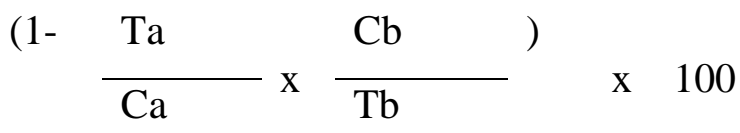

Where,

$\mathrm{Ta}=$ Number of mites on treated trees after acaricidal application

$\mathrm{Tb}=$ Number of mites on treated trees before acaricidal application

$\mathrm{Ca}=$ Number of mites on untreated trees after acaricidal application

$\mathrm{Cb}=$ Number of mites on untreated trees before acaricidal application 


\section{Results and Discussion}

\section{Bio efficacy against ERM during 2018-19}

Perusal of the data presented in table 1 reveal that all the acaricidal treatments were better than the untreated control. Exhibited data reveal that at Nagbal cumulative mean percent mortality of 61.59 was recorded in Etoxazole @ $0.2 \mathrm{ml}$ water $^{-1}$ followed by 63.96 and 66.03 @ $0.5 \mathrm{ml}$ and $0.8 \mathrm{ml}$ water $^{-1}$ respectively. Highest cumulative mean percent mortality of 70.62 was observed in concentration of $1.1 \mathrm{ml}$ water $^{-1}$. However, standard check (Propargite) @ 2.0ml water ${ }^{-1}$ recorded the overall highest cumulative mean percent mortality of 72.80 . Water sprayed plants (control) recorded the lowest cumulative mean percent mortality of 12.28. Maximum and minimum yield of $\mathrm{A}$ grade apples (boxes/tree) of 4.30 and 3.05 was recorded in concentration of $1.1 \mathrm{ml}$ and $0.2 \mathrm{ml}$ water $^{-1}$ respectively. Propargite @ $2 \mathrm{ml}$ water $^{-1}$ recorded the yield of 4.25 boxes of A grade apples/tree. In Chandpora cumulative mean percent mortality of 62.70 was recorded in Etoxazole @ 0.2 ml water ${ }^{-1}$ followed by 65.10 and 67.01 in concentrations of $0.5 \mathrm{ml}$ and $0.8 \mathrm{ml}$ water $^{-1}$ respectively. Highest cumulative mean percent mortality of 70.0 was observed in concentration of $1.1 \mathrm{ml} \mathrm{water}^{-1}$. Propargite @ $2 \mathrm{ml} \mathrm{water}^{-1}$ which was used as standard check recorded highest cumulative mean percent mortality of 73.34. Water sprayed plants (control) recorded the lowest cumulative mean percent mortality of 18.59 . Maximum and minimum yield of $\mathrm{A}$ grade apples (boxes/tree) of 4.35 and 3.35 was recorded in concentration of $1.1 \mathrm{ml}$ and $0.2 \mathrm{ml}$ water ${ }^{-1}$ respectively. Propargite @ $2 \mathrm{ml}$ water $^{-1}$ recorded the yield of 4.53 boxes of A grade apples/tree. All the treatments were significantly different from untreated control, when tested at $\mathrm{P}=0.05$. The order of efficacy was observed as Propargite 57 EC @ $2.0 \mathrm{ml}>$ Etoxazole $10 \mathrm{SC} @>1.1 \mathrm{ml}>0.8 \mathrm{ml}>0.5 \mathrm{ml}>$ $0.2 \mathrm{ml}$ water $^{-1}$.

Table.1 Bio efficacy of Etoxazole 10 SC against European red mite (Panonychus ulmi) at Nagbal and Chandpora (District Ganderbal and Srinagar) Kashmir during 2018-19

\begin{tabular}{|c|c|c|c|c|c|c|c|c|c|c|}
\hline \multicolumn{11}{|c|}{ Nagbal } \\
\hline \multirow[t]{2}{*}{ Treatments } & \multirow{2}{*}{$\begin{array}{c}\text { Dose } \\
\text { water }^{-1}\end{array}$} & \multirow{2}{*}{$\begin{array}{c}\text { Pre } \\
\text { treatment } \\
\text { count } / 20 \\
\text { leaves }\end{array}$} & \multicolumn{6}{|c|}{ Percent Mortality (Days after treatment) } & \multirow{2}{*}{$\begin{array}{c}\text { Cumulative } \\
\text { mean } \\
\text { percent } \\
\text { mortality }\end{array}$} & \multirow{2}{*}{$\begin{array}{c}* \text { Yield of } \\
\text { A grade } \\
\text { apples/tree }\end{array}$} \\
\hline & & & 3 & 7 & 10 & 14 & 21 & 28 & & \\
\hline \multirow[t]{4}{*}{ Etoxazole $10 \mathrm{SC}$} & $0.2 \mathrm{ml}$ & 33.17 & 35.96 & 46.13 & 55.98 & 68.44 & 77.98 & 89.93 & 61.59 & 3.05 \\
\hline & $0.5 \mathrm{ml}$ & 33.33 & 37.45 & 47.40 & 59.10 & 69.30 & 79.30 & 93.05 & 63.96 & 3.30 \\
\hline & $0.8 \mathrm{ml}$ & 32.50 & 39.35 & 48.21 & 60.00 & 70.36 & 80.74 & 93.97 & 66.03 & 3.55 \\
\hline & $1.1 \mathrm{ml}$ & 33.10 & 41.87 & 50.94 & 64.87 & 74.00 & 80.38 & 94.73 & 70.62 & 4.30 \\
\hline Propargite 57 EC & $2 \mathrm{ml}$ & 32.90 & 48.96 & 53.41 & 68.35 & 80.15 & 88.25 & 97.70 & 72.80 & 4.25 \\
\hline Control & - & 30.67 & 5.15 & 8.80 & 11.02 & 13.19 & 16.61 & 18.91 & 12.28 & 2.08 \\
\hline C.D $(P \leq 0.05)$ & & & 1.50 & 1.48 & 1.55 & 1.87 & 1.75 & 2.04 & & \\
\hline \multicolumn{11}{|c|}{ Chandpora } \\
\hline \multirow[t]{4}{*}{ Etoxazole $10 \mathrm{SC}$} & $0.2 \mathrm{ml}$ & 34.34 & 35.53 & 44.27 & 60.97 & 69.61 & 77.18 & 88.63 & 62.70 & 3.35 \\
\hline & $0.5 \mathrm{ml}$ & 34.34 & 37.46 & 47.07 & 63.19 & 71.74 & 79.12 & 92.04 & 65.10 & 3.55 \\
\hline & $0.8 \mathrm{ml}$ & 32.44 & 39.88 & 48.42 & 64.48 & 73.23 & 80.65 & 95.37 & 67.01 & 3.70 \\
\hline & $1.1 \mathrm{ml}$ & 25.37 & 42.40 & 49.81 & 67.55 & 78.74 & 88.01 & 96.52 & 70.00 & 4.35 \\
\hline Propargite 57 EC & $2 \mathrm{ml}$ & 20.18 & 52.16 & 58.93 & 69.71 & 78.77 & 87.32 & 93.31 & 73.34 & 4.53 \\
\hline Control & - & 32.67 & 9.97 & 15.60 & 17.34 & 20.09 & 23.73 & 24.84 & 18.59 & 2.05 \\
\hline C.D $(P \leq 0.05)$ & & & 1.45 & 1.33 & 1.82 & 1.99 & 1.80 & 1.69 & & \\
\hline
\end{tabular}

$*=$ Boxes/trees 
Table.2 Bio efficacy of Etoxazole 10 SC against two spotted spider mite (Tetranychus ulmi) at Nagbal and Chandpora (District Ganderbal and Srinagar) Kashmir during 2018-19

\begin{tabular}{|c|c|c|c|c|c|c|c|c|c|c|c|}
\hline \multicolumn{12}{|c|}{ Nagbal } \\
\hline \multirow[t]{2}{*}{ Treatments } & \multirow{2}{*}{$\begin{array}{c}\text { Dose } \\
\text { water }^{-1}\end{array}$} & \multirow{2}{*}{$\begin{array}{l}\text { Pre } \\
\text { treatment } \\
\text { count } / 20 \\
\text { leaves }\end{array}$} & \multicolumn{6}{|c|}{ Percent Mortality (Days after treatment) } & \multicolumn{2}{|c|}{$\begin{array}{l}\text { Cumulative } \\
\text { mean } \\
\text { percent } \\
\text { mortality }\end{array}$} & \multirow{2}{*}{$\begin{array}{l}\text { Yield of } A \\
\text { grade } \\
\text { apples/tree }\end{array}$} \\
\hline & & & 3 & 7 & 10 & 14 & 21 & 28 & & & \\
\hline \multirow[t]{4}{*}{ Etoxazole $10 \mathrm{SC}$} & $0.2 \mathrm{ml}$ & 17.65 & 32.96 & 42.01 & 46.66 & 56.56 & 71.58 & 86.59 & \multicolumn{2}{|c|}{56.06} & 3.05 \\
\hline & $0.5 \mathrm{ml}$ & 18.17 & 43.49 & 48.62 & 53.18 & 65.40 & 78.96 & 91.46 & \multicolumn{2}{|c|}{63.52} & 3.30 \\
\hline & $0.8 \mathrm{ml}$ & 18.27 & 44.21 & 49.91 & 55.20 & 66.65 & 82.11 & 92.79 & \multicolumn{2}{|c|}{65.14} & 3.55 \\
\hline & $1.1 \mathrm{ml}$ & 17.61 & 46.16 & 54.09 & 59.32 & 72.77 & 87.65 & 94.65 & \multicolumn{2}{|c|}{69.11} & 4.30 \\
\hline Propargite 57 EC & $2 \mathrm{ml}$ & 12.49 & 48.98 & 56.69 & 70.70 & 77.63 & 87.27 & 92.69 & \multicolumn{2}{|c|}{72.32} & 4.25 \\
\hline Control & - & 18.42 & 4.69 & 7.12 & 12.35 & 16.32 & 20.10 & 22.61 & \multicolumn{2}{|c|}{13.86} & 2.08 \\
\hline C.D $(P \leq 0.05)$ & & & 1.22 & 1.37 & 0.87 & 1.77 & 0.87 & 1.37 & & & \\
\hline \multicolumn{12}{|c|}{ Chandpora } \\
\hline \multirow[t]{4}{*}{ Etoxazole $10 \mathrm{SC}$} & $0.2 \mathrm{ml}$ & 17.40 & 31.03 & 41.76 & 50.57 & 59.38 & 70.31 & 87.55 & \multicolumn{2}{|c|}{56.77} & 3.35 \\
\hline & $0.5 \mathrm{ml}$ & 17.99 & 36.23 & 45.31 & 58.29 & 64.96 & 76.45 & 90.92 & \multicolumn{2}{|c|}{62.02} & 3.55 \\
\hline & $0.8 \mathrm{ml}$ & 18.24 & 40.21 & 48.80 & 61.23 & 70.56 & 82.81 & 92.50 & \multicolumn{2}{|c|}{66.02} & 3.70 \\
\hline & $1.1 \mathrm{ml}$ & 17.50 & 42.91 & 55.94 & 63.21 & 71.80 & 85.90 & 95.27 & \multicolumn{2}{|c|}{69.17} & 4.35 \\
\hline Propargite 57 EC & $2 \mathrm{ml}$ & 18.24 & 44.95 & 59.43 & 64.98 & 74.58 & 87.16 & 96.71 & \multicolumn{2}{|c|}{71.58} & 4.53 \\
\hline Control & - & 18.07 & 6.80 & 9.56 & 13.07 & 17.50 & 21.19 & 22.48 & \multicolumn{2}{|c|}{15.10} & 2.05 \\
\hline C.D $(P \leq 0.05)$ & & & 1.76 & 1.78 & 1.64 & 1.91 & 1.39 & 1.475 & & & \\
\hline \multicolumn{12}{|c|}{$*=$ Boxes $/$ trees } \\
\hline \multicolumn{12}{|c|}{$\begin{array}{c}\text { Table.3 Toxicity of Etoxazole } 10 \text { SC against Amblyseius population on apple cv. Red Delicious } \\
\text { at Nagbal, Ganderbal during 2018-19 }\end{array}$} \\
\hline \multirow[t]{2}{*}{ Treatments } & $\begin{array}{c}\text { Dose } \\
\text { water }^{-1}\end{array}$ & \multirow{2}{*}{\multicolumn{2}{|c|}{$\begin{array}{c}\text { *Pre } \\
\text { treatment } \\
\text { count/5 leaves }\end{array}$}} & \multicolumn{6}{|c|}{ *Post treatment count } & \multicolumn{2}{|c|}{$\begin{array}{l}\text { Cumulative } \\
\text { mean percent } \\
\text { mortality }\end{array}$} \\
\hline & & & & 1 & & 3 & 7 & & 15 & & \\
\hline $\begin{array}{c}\text { Etoxazole } 10 \\
\text { SC }\end{array}$ & $0.2 \mathrm{ml}$ & 1.50 & & $\begin{array}{c}1.42 \\
(13.80)\end{array}$ & & $\begin{array}{l}.30 \\
2.86)\end{array}$ & $\begin{array}{r}1.16 \\
(26.57\end{array}$ & & $\begin{array}{c}1.10 \\
(17.23)\end{array}$ & & 20.11 \\
\hline & $0.5 \mathrm{ml}$ & 1.60 & & $\begin{array}{c}1.45 \\
(18.30)\end{array}$ & & $\begin{array}{l}.31 \\
3.93)\end{array}$ & $\begin{array}{r}1.21 \\
(31.52\end{array}$ & & $\begin{array}{c}1.08 \\
(20.81)\end{array}$ & & 23.64 \\
\hline & $0.8 \mathrm{ml}$ & 1.62 & & $\begin{array}{c}1.46 \\
(21.31)\end{array}$ & & $\begin{array}{l}.28 \\
8.84)\end{array}$ & $\begin{array}{r}1.15 \\
(32.26\end{array}$ & & $\begin{array}{c}1.10 \\
(23.15)\end{array}$ & & 26.39 \\
\hline & $1.1 \mathrm{ml}$ & 1.78 & & $\begin{array}{c}1.57 \\
(18.99)\end{array}$ & & $\begin{array}{l}.44 \\
1.45)\end{array}$ & $\begin{array}{r}1.21 \\
(35.27\end{array}$ & & $\begin{array}{c}1.15 \\
(24.34)\end{array}$ & & 27.51 \\
\hline $\begin{array}{c}\text { Propargite } \\
57 \text { EC }\end{array}$ & $2 \mathrm{ml}$ & $\begin{array}{r}1.72 \\
(4.06\end{array}$ & & $\begin{array}{c}1.65 \\
(23.32)\end{array}$ & & $\begin{array}{l}.32 \\
2.92)\end{array}$ & $\begin{array}{r}1.16 \\
(42.15\end{array}$ & & $\begin{array}{c}1.00 \\
(25.69)\end{array}$ & & 31.02 \\
\hline Control & -- & $\begin{array}{r}1.64 \\
(2.12\end{array}$ & & $\begin{array}{c}1.60 \\
(3.96)\end{array}$ & & $\begin{array}{l}.57 \\
.02)\end{array}$ & $\begin{array}{r}1.52 \\
(11.02\end{array}$ & & $\begin{array}{l}1.46 \\
(6.03)\end{array}$ & & 7.00 \\
\hline C.D $(P \leq 0.05)$ & & & & 1.61 & & .61 & 1.52 & & 1.74 & & \\
\hline
\end{tabular}


Table.4 Toxicity of Etoxazole 10 SC against Amblyseius and Coccinellid populations on apple cv. Red Delicious at Chandpora, Srinagar during 2018-19

\begin{tabular}{|c|c|c|c|c|c|c|c|c|c|c|c|c|c|}
\hline \multirow[t]{3}{*}{ Treatments } & \multirow[t]{3}{*}{$\begin{array}{c}\text { Dose } \\
\text { water }^{-1}\end{array}$} & \multicolumn{2}{|c|}{$\begin{array}{c}* \text { Pre } \\
\text { treatment } \\
\text { count } / 5 \\
\text { leaves }\end{array}$} & \multicolumn{8}{|c|}{ *Post treatment count } & \multicolumn{2}{|c|}{$\begin{array}{c}\text { Cumulative } \\
\text { mean } \\
\text { percent } \\
\text { mortality }\end{array}$} \\
\hline & & \multirow[t]{2}{*}{$\mathbf{A}$} & \multirow[t]{2}{*}{$\mathbf{C}$} & \multicolumn{4}{|c|}{$\mathbf{A}$} & \multicolumn{4}{|c|}{$\mathbf{C}$} & \multirow[t]{2}{*}{$\mathbf{A}$} & \multirow[t]{2}{*}{ C } \\
\hline & & & & 1 & 3 & 7 & 15 & 1 & 3 & 7 & 15 & & \\
\hline \multirow[t]{4}{*}{$\begin{array}{c}\text { Etoxazole } 10 \\
\text { SC }\end{array}$} & $0.2 \mathrm{ml}$ & 1.57 & 1.12 & $\begin{array}{c}1.40 \\
(10.72)\end{array}$ & $\begin{array}{c}1.30 \\
(4.50)\end{array}$ & $\begin{array}{c}1.22 \\
(22.10)\end{array}$ & $\begin{array}{c}1.13 \\
27.76)\end{array}$ & $\begin{array}{c}1.05 \\
(7.14)\end{array}$ & $\begin{array}{c}0.98 \\
(12.93)\end{array}$ & $\begin{array}{c}0.86 \\
(23.58)\end{array}$ & $\begin{array}{c}0.76 \\
(32.46)\end{array}$ & 19.32 & 19.02 \\
\hline & $0.5 \mathrm{ml}$ & 1.51 & 1.10 & $\begin{array}{c}1.32 \\
(12.39)\end{array}$ & $\begin{array}{c}1.24 \\
(4.22)\end{array}$ & $\begin{array}{c}1.13 \\
(24.75)\end{array}$ & $\begin{array}{c}1.02 \\
32.57)\end{array}$ & $\begin{array}{c}1.01 \\
(8.34)\end{array}$ & $\begin{array}{c}0.87 \\
(20.68)\end{array}$ & $\begin{array}{c}0.77 \\
(\mathbf{3 0 . 0 7 )}\end{array}$ & $\begin{array}{c}0.72 \\
(35.48)\end{array}$ & 22.02 & 23.64 \\
\hline & $0.8 \mathrm{ml}$ & 1.53 & 1.26 & $\begin{array}{c}1.30 \\
(15.19)\end{array}$ & $\begin{array}{c}1.18 \\
(2.64)\end{array}$ & $\begin{array}{c}1.10 \\
(27.54)\end{array}$ & $\begin{array}{c}0.97 \\
35.96)(\end{array}$ & $\begin{array}{c}1.10 \\
(12.98)\end{array}$ & $\begin{array}{c}0.99 \\
(21.72)\end{array}$ & $\begin{array}{c}0.87 \\
(32.01)\end{array}$ & $\begin{array}{c}0.76 \\
(40.87)\end{array}$ & 25.33 & 26.90 \\
\hline & $1.1 \mathrm{ml}$ & 1.53 & 1.13 & $\begin{array}{c}1.30 \\
(14.77)\end{array}$ & $\begin{array}{c}1.17 \\
(5.21)\end{array}$ & $\begin{array}{c}1.07 \\
(30.08)\end{array}$ & $\begin{array}{l}0.96 \\
37.23)(\end{array}$ & $\begin{array}{c}1.01 \\
(10.97)\end{array}$ & $\begin{array}{c}0.86 \\
(24.47)\end{array}$ & $\begin{array}{c}0.71 \\
(38.79)\end{array}$ & $\begin{array}{c}0.64 \\
(45.38)\end{array}$ & 26.30 & 29.90 \\
\hline $\begin{array}{c}\text { Propargite } 57 \\
\text { EC }\end{array}$ & $2 \mathrm{ml}$ & 1.72 & 1.14 & $\begin{array}{c}1.45 \\
(15.78)\end{array}$ & $\begin{array}{c}1.32 \\
(3.34)\end{array}$ & $\begin{array}{c}1.20 \\
(30.30)\end{array}$ & $\begin{array}{c}0.98 \\
43.09)(\end{array}$ & $\begin{array}{c}0.95 \\
(17.20)\end{array}$ & $\begin{array}{c}0.85 \\
(26.04)\end{array}$ & $\begin{array}{c}0.74 \\
(36.02)\end{array}$ & $\begin{array}{c}0.66 \\
(43.82)\end{array}$ & 28.13 & 30.77 \\
\hline Control & -- & 1.59 & 1.07 & $\begin{array}{c}1.55 \\
(2.69)\end{array}$ & $\begin{array}{c}1.53 \\
(1.76)\end{array}$ & $\begin{array}{c}1.49 \\
(6.51)\end{array}$ & $\begin{array}{c}1.45 \\
(8.70)\end{array}$ & $\begin{array}{c}1.06 \\
(1.88)\end{array}$ & $\begin{array}{c}1.04 \\
(3.76)\end{array}$ & $\begin{array}{c}1.00 \\
(7.00)\end{array}$ & $\begin{array}{c}0.95 \\
(11.66)\end{array}$ & 5.45 & 6.07 \\
\hline C.D $(P \leq 0.05)$ & & & & 1.47 & 1.84 & 1.06 & 1.31 & 1.49 & 0.95 & 1.45 & 1.38 & & \\
\hline
\end{tabular}

Percent mortality in parenthesis; * Mean of three replications; $\mathrm{A}=$ Amblyseius $\mathrm{C}=$ Coccinelid

Bio efficacy against two spotted spider mite during 2018-19

The data presented in table 2 reveal that all the acaricidal treatments were better than the untreated control. Observed data show that at Nagbal lowest cumulative mean percent mortality of 56.06 was recorded in Etoxazole @ $0.2 \mathrm{ml} \mathrm{water}^{-1}$ followed by 63.52 and 65.14 @ $0.5 \mathrm{ml}$ and $0.8 \mathrm{ml}$ water $^{-1}$ respectively. Highest cumulative mean percent mortality of 69.11 was observed in concentration of $1.1 \mathrm{ml}$ water $^{-1}$. Propargite which was used as a standard check@2.0mlwater ${ }^{-1}$ recorded the overall highest cumulative mean percent mortality of 72.32. Water sprayed plants (control) recorded the lowest cumulative mean percent mortality of 13.86 . Maximum and minimum yield of $\mathrm{A}$ grade apples (boxes/tree) of 4.30 and 3.05 was recorded in concentration of $1.1 \mathrm{ml}$ and $0.2 \mathrm{ml}$ water $^{-1}$ respectively. Propargite @ $2 \mathrm{ml}$ water $^{-1}$ recorded the yield of 4.25 boxes of A grade apples/tree. In Chandpora cumulative mean percent mortality of 56.77 was recorded in Etoxazole @ 0.2 ml water ${ }^{-1}$ followed by 62.02 and 66.02 in concentrations of $0.5 \mathrm{ml}$ and $0.8 \mathrm{ml}$ water $^{-1}$ respectively. Highest cumulative mean percent mortality of 69.17 was observed in concentration of $1.1 \mathrm{ml}^{\text {water }}{ }^{-}$ 1. Propargite (standard check) @ 2ml water ${ }^{-1}$ recorded highest cumulative mean percent mortality of 71.58. Water sprayed plants (control) recorded the lowest cumulative mean percent mortality of 15.10 . Maximum and minimum yield of A grade apples (boxes/tree) of 4.35 and 3.35 was recorded in concentration of $1.1 \mathrm{ml}$ and $0.2 \mathrm{ml}$ water $^{-1}$ 
respectively. Propargite @ $2 \mathrm{ml}$ water $^{-1}$ recorded the yield of 4.53 boxes of $\mathrm{A}$ grade apples/tree. All the treatments were significantly different from untreated control, when tested at $\mathrm{P}=0.05$. The order of efficacy was observed as Propargite 57 EC @ $2.0 \mathrm{ml}>$ Etoxazole $10 \mathrm{SC} @>1.1 \mathrm{ml}>0.8 \mathrm{ml}>0.5 \mathrm{ml}>$ $0.2 \mathrm{ml} \mathrm{water}^{-1}$.

No phytotoxic effect like leaf injury yellowing, wilting, necrosis, hyponasty and epinasty on apple leaves were observed in any of the concentrations tested and there were no apparent phytotoxicity symptoms during the study programme at both the locations during the year 2018-19.

\section{Toxicity to natural enemies at Nagbal district Ganderbal during 2018-19}

Toxicity of Etoxazole was evaluated against Amblyseius associated with ERM and TSSM at Nagbal, Ganderbal (table 3). Cumulative mean percent mortality of Amblyseius was recorded as 27.51 against Etoxazole @ 1.1ml water $^{-1}$ followed by 26.39 and $23.64 @ 0.8 \mathrm{ml}$ and $0.5 \mathrm{ml}$ water $^{-1}$ respectively. Lowest cumulative mean percent mortality of Amblyseius against Etoxazole was recorded as 20.11 @ $0.2 \mathrm{ml}$ water 1 . Propargite used as standard check recorded highest cumulative mean percent mortality of Amblyseius as $31.02 @ 2.0 \mathrm{ml}$ water $^{-1}$. Water resulted in the lowest cumulative mean percent mortality of 7.0. The order of toxicity was observed as Propargite 57 EC @ $2.0 \mathrm{ml}>$ Etoxazole 10 SC@ $>1.1 \mathrm{ml}>0.8 \mathrm{ml}>0.5 \mathrm{ml}>0.2 \mathrm{ml}$ water 1

\section{Toxicity to natural enemies at Chandpora district Srinagar during 2018-19}

Perusal of the data in table 4, Etoxazole was evaluated against two important natural enemies, Amblyseius and Coccinellids associated with ERM and TSSM in the
Kashmir Valley. Highest cumulative mean percent mortality of Amblyseius was recorded as 26.30 against Etoxazole @ 1.1 ml water $^{-1}$ followed by 25.33 and $22.02 @ 0.8 \mathrm{ml}$ and $0.5 \mathrm{ml}$ water $^{-1}$ respectively. Lowest cumulative mean percent mortality of Amblyseius against Etoxazole was recorded as 19.32 @ $0.2 \mathrm{ml}$ water $^{-1}$. Propargite used as standard check recorded highest cumulative mean percent mortality of Amblyseius as 28.13@2.0ml water $^{-1}$. Water resulted in the lowest cumulative mean percent mortality of 5.45.

Highest cumulative mean percent mortality of Coccinellid was recorded as 29.90 against Etoxazole@1.1 ml water ${ }^{-1}$ followed by 26.90 and 23.64 @ $0.8 \mathrm{ml}$ and $0.5 \mathrm{ml}$ water $^{-1}$ respectively. Lowest cumulative mean percent mortality of Coccinellid against Etoxazole was recorded as $19.02 @ 0.2 \mathrm{ml}^{\text {water }}{ }^{-1}$. Propargite used as standard check recorded highest cumulative mean percent mortality of Coccinellid as $30.77 @ 2.0 \mathrm{ml}$ water $^{-1}$. Water resulted in the lowest cumulative mean percent mortality of 6.07 . The order of toxicity was observed as Propargite 57 EC @ $2.0 \mathrm{ml}>$ Etoxazole $10 \mathrm{SC} @>1.1 \mathrm{ml}>0.8 \mathrm{ml}$ $>0.5 \mathrm{ml}>0.2 \mathrm{ml}$ water ${ }^{-1}$. These results are in agreement with Sherwani et,al., 2017, Sahu et,al., 2008 and Bhanu et,al., 2006 who observed that with increase in dose of acaricides the mortality of mite pests as well as that of natural enemies also increases manifold.

\section{References}

Anonymous, 2014. Management of pests on apple in Kashmir. Division of Entomology, Directorate of Extension education, SKUAST-K, Shalimar.

Bhalla, O.P. and Gupta, P.R. 1993. Insect pest of temperate fruits. Advances in Horticulture - Fruit Crops Malhotra Publishing House, New Delhi, India.,3: 1557-1589 
Bhanu, K. V., Reddy, P. S. and Zeheruddeen, S. M. 2006. Evaluation of some acaricide against leaf mite and sheath mite in rice. Indian Journal of Plant Protection, 34 (1): 132-133

Henderson CF, Tilton EW. 1955. Tests with acaricides against brown wheat mite. Journal of Economic Entomology 48(2):157-161.

Madson, H. and Morgan, C.V.G. (1970) Pome fruit pest's ad their control. Journal of Entomological Sciences, $37: 41-45$
Sahu, B. Chakraborty, G., and Somachoudhary, A. K. 2008. Efficacy of Spiromesifen $240 \mathrm{SC}$ in the management of tea red spider mite (Oligoonychus coffee Niether) on tea. Environment and Ecology 24(1)

Sherwani. A, Mukhtar, Malik, Sofi, Muneer Ahmad and Saima Maqsood. 2017. Field Evaluation of New Ovicidal Acaricide (Tafethion) against Two Major Insect Pests of Apple in Kashmir. Int.J.Curr.Microbiol.App.Sci. $\quad$ 6(7): 3905-3916.

\section{How to cite this article:}

Asma Sherwani, Peerzada Shafat Hussian and Malik Mukhtar. 2020. Bio Efficacy and Effect on Natural Enemies of Acaricide Etoxazole 10 SC against Mite Pests of Apple in Kashmir. Int.J.Curr.Microbiol.App.Sci. 9(02): 2986-2992. doi: https://doi.org/10.20546/ijcmas.2020.902.341 